\title{
Verification of equipment for measuring the diffusion capacity of the lung for carbon monoxide
}

\section{To the Editors:}

In the paper by JENSEN et al. [1] and the accompanying editorial by FARRÉ and NAVAJAS [2] the importance of quality assurance in the respiratory physiology laboratory is highlighted.

The JENSEN et al. [1] study represents a tremendous and successful effort on quality assurance and quality control as part of a large clinical study. The study or probably more likely the presentation of the study by JENSEN et al. [1] has a few limitations seen from a metrological point of view to which I want to draw attention. Traceability is the key stone of quality control of measurements and must be addressed in all metrological studies. Metrological traceability is a property of a measurement result relating the result to a stated metrological reference through an unbroken chain of calibrations of a measuring system or comparisons, each contributing to the stated measurement uncertainty [3]. Thus, traceability refers to the completeness of the information about every step in a process chain.

In the respiratory physiology laboratory we depend on measurements with specified accuracy. Our basic measurements are volume, time, flow, pressure, concentrations, mass, length, temperature and humidity. It is within the limits of respiratory physiology laboratories to verify most of these measurements internally, but many respiratory laboratories, if any, are not able to verify gas concentration with sufficient accuracy and precision (fig. 1). Therefore a clear agreement should be developed with the supplier on the methods by which conformance to purchaser's requirements will be verified [4]. In the JENSEN et al. [1] study this problem is obvious but most of us cannot perform an internal verification of our carbon monoxide and tracer gas and we have to rely on the supplier's verification. That's why this issue should be addressed carefully.

In the JENSEN et al. [1] paper, the traceability of the gas concentration and volume measurements used for simulation are not stated. A reference is given to the description of the methods in a previous paper by JENSEN et al. [5] in which the gas is described as a "precision gas" and the volumes are delivered by "precision syringes". The vital importance of gas concentration accuracy and gas volume accuracy in the diffusing capacity of the lung for carbon monoxide $(D \mathrm{~L}, \mathrm{CO})$ simulation study is due to the working principle of the $D \mathrm{~L}, \mathrm{CO}$ simulator which delivers the gas volumes and concentrations to be measured by the laboratory $[1,5]$. If the gas concentrations (fig. 1) or volumes are not accurate within specified limits the simulations carries no validity.

To raise the questions above might seem pedantic, but as stated in the accompanying editorial the devil is in the detail [2] and traceability is not reported or overlooked to often.

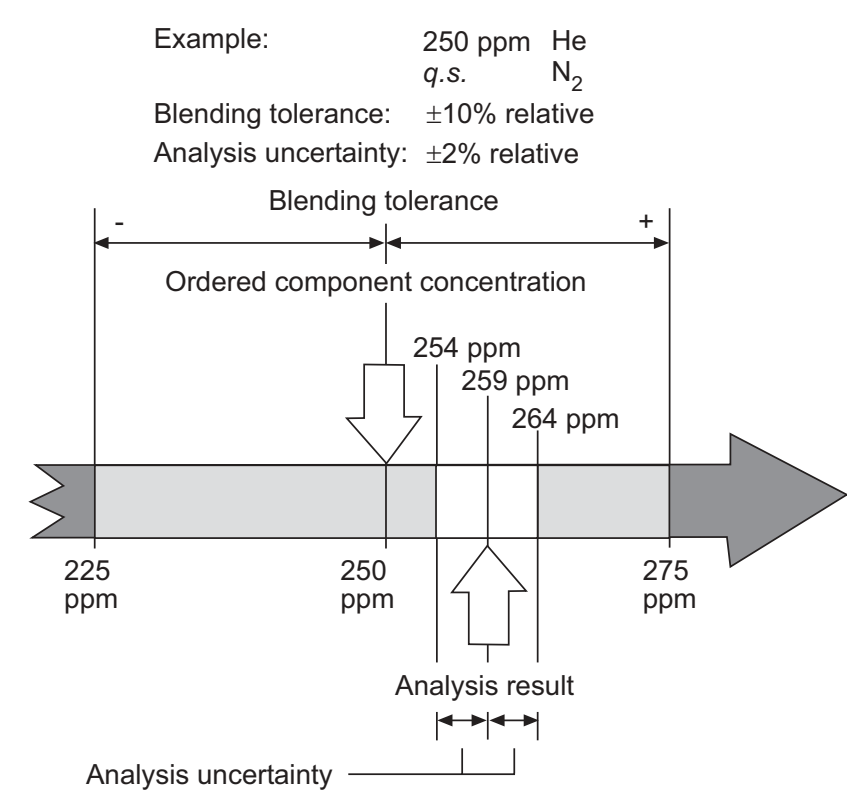

FIGURE 1. Concentration of gas $(\mathrm{He})$ in $\mathrm{N}_{2}$. The blending tolerance is the maximum difference between the ordered concentration and the delivered concentration (in mixture). Analyses uncertainty is the maximum difference between the analyses result and the true concentration and in the example reported relative to the analysed component concentration. q.s.: quantum satis. The figure is printed with permission from AGA A/S, Linde Healthcare (Copenhagen, Denmark).

The main reason for asking the questions above is not a real concern about the validity of the results, but the chance to get source information on accuracy of volume and gas concentration measurements, not easily obtainable elsewhere. The Salt Lake City laboratory (Salt Lake City, UT, USA) is highly reputed for decades of standardisation of flow measurements and has been a role model for many of us trying to improve the quality of volume and flow measurement. Therefore, we have a chance to have the difficult questions on traceability of syringe volume and gas concentration verification answered.

I hope that this type of positive industry-driven quality control will spread from industry to our clinical work now the simulator has become commercially available.

\section{F. Madsen}

Correspondence: F. Madsen, Allergy and Lung Clinic, Sct Olaigade 39, Elsinore 3000, Denmark. E-mail: flem-mad@dadlnet. dk

Statement of Interest: None declared. 


\section{REFERENCES}

1 Jensen R, Leyk M, Crapo R, et al. Quality control of DL,CO instruments in global clinical trials. Eur Respir J 2009; 33: 828-834.

2 Farré R, Navajas D. Quality control: a necessary, but sometimes overlooked, tool for improving respiratory medicine. Eur Respir J 2009; 33: 722-723.

3 ISO/IEC Guide 99:2007. International vocabulary of metrology basic and general concepts and associated terms (VIM). Geneva, International Organization for Standardization, 2007.
4 ISO 9004:2000. Quality management systems - guidelines for performance improvements. Geneva, International Organization for Standardization, 2000.

5 Jensen RL, Teeter JG, England RD, et al. Instrument accuracy and reproducibility in measurements of pulmonary function. Chest 2007, 132: 388-395.

\section{Infective complications from endobronchial ultrasound- transbronchial needle aspiration}

\section{To the Editors:}

We read with significant interest the report from HAAs [1] in a recent issue of the European Respiratory Journal, which described two cases of infectious complications following endobronchial ultrasound-guided transbronchial needle aspiration (EBUS-TBNA). This technique has been widely adopted for the evaluation of mediastinal and hilar lesions for several reasons, including its high-diagnostic accuracy, minimally invasive nature and excellent safety profile. As stated by HAAS [1], no significant complications have previously been reported.

We recently experienced a similar complication in our interventional bronchoscopy centre (Royal Melbourne Hospital, Parkville, Australia). A female with small cell lung cancer and a moderate-sized retrosternal thyroid lesion underwent EBUS-TBNA sampling of the thyroid lesion for the purpose of staging. EBUS imaging demonstrated a cystic space and cytology performed on the TBNA specimen revealed normal thyroid colloid. She presented to her local medical officer with erythema over the sternal notch $48 \mathrm{~h}$ after the procedure. Despite initial therapy with flucloxacillin this progressed and on re-presentation to our centre she had developed a spontaneous purulent discharge from this site, with subsequent resolution over the following 6 days. Penicillin-sensitive Streptococcus pneumoniae was isolated from multiple culture specimens of purulent material.

Bacteraemia following conventional TBNA is extremely rare [2]. The sole description of bacteraemia following TBNA reported Streptococcus viridans as the causative agent, suggesting an oral source [3]. Patients described by HAAs [1] and EPSTEIN et al. [4] developed bacterial infections complicating TBNA with microbiological and clinical features, highly consistent with inoculation of oropharyngeal bacteria into the relatively avascular pericardial space $[1,4]$. Infection resulting from direct inoculation of relatively avascular necrotic tissue following endoscopic fine needle aspiration has also been reported [5]. With respect to our patient, S. pneumoniae is a common nasopharyngeal commensal and we believe infection was similarly caused by bronchoscope contamination during insertion through the upper airway, with subsequent direct inoculation of bacteria by the TBNA needle into an avascular cystic space.

EBUS-TBNA appears to be the optimal procedure for sampling of mediastinal and hilar lesions due to its safety in comparison to formal surgical procedures. However, our experience, and that of HAAS [1], indicates that a complication rate of $0 \%$ for a novel technique almost certainly means insufficient procedures have been performed to fully inform commentary on complications rates, or possibly that complications remain unreported. It is important for proceduralists performing EBUS-TBNA to be aware that, while rare, significant infection is a recognised complication. Contamination of the bronchoscope working channel during introduction into the airway is possible and direct inoculation of bacteria appears the likely cause of infective complications $[1,4,5]$.

We commend HAAS [1] for the timely report on the potential for infection complicating EBUS-TBNA, but feel the issue of antibiotic selection in such cases should be emphasised. Further studies are required to elucidate the organisms involved in infections following EBUS-TBNA. However, our experience, and that reported by HAAS [1], suggests empiric antibiotic use in patients with clinical suspicion of infection must include agents with activity against indigenous oral and nasopharyngeal organisms. Prophylactic antibiotics for EBUSTBNA do not appear to be indicated on the basis of risk of bacteraemia; however, we feel our experience, and previously reported experiences $[1,4,5]$, indicate further evaluation is required to determine the role of prophylactic antibiotics in specific patient groups. Consideration of use of antibiotic prophylaxis may be appropriate in patients with relatively avascular lesions such as cysts or necrotic lymph nodes, or in immunocompromised patients at high risk of local infective complications.

\section{D.P. Steinfort*, D.F. Johnson ${ }^{\#}$ and L.B. Irving* \\ *Dept of Respiratory Medicine, Royal Melbourne Hospital, Parkville, and "Dept of Infectious Diseases, Austin Hospital, Heidelberg, Australia.}

